\section{Sterben und Tod}

Gabriel Looser

Was in uns nicht stirbt -

Erfahrungen der Unsterblichkeit

München: Kösel-Verlag; 2008.

256 Seiten. Fr. 31.90

ISBN 978-3-466-36791-7

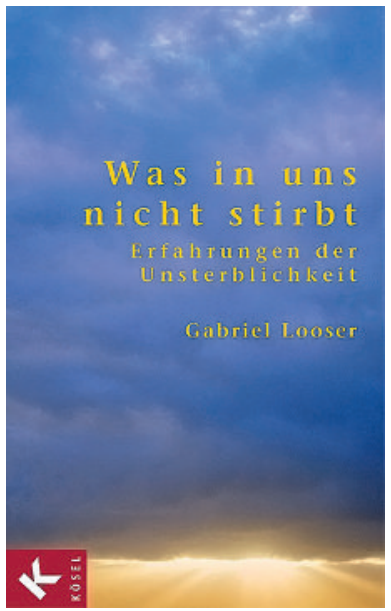

Wer sich durch den obigen Titel irritiert fühlt, lese vielleicht besser nicht weiter, möge sich aber doch die Frage stellen, was denn daran eigentlich das Irritierende oder gar das Angstauslösende sei. Vielen dürfte schon nur die Formulierung «Erfahrungen der Unsterblichkeit» als paradox, wenn nicht gar - für unsere übliche Art des Denkens - als grundsätzlich unmöglich auffallen.

Der Buchautor Dr. Gabriel Looser ist Theologe und Psychologe. Er besitzt eine 12-jährige reiche Erfahrung als Spital-Seelsorger von Kranken und Sterbenden sowie deren Angehörigen. Seit mehreren Jahren leitet er ein Institut zur spirituellen Sterbebegleitung in Bern. Zusätzliche Impulse erhielt er durch Begegnungen mit Lehrern des tibetischen Buddhismus und der Sufi-Tradition. Erwähnen möchte ich noch, dass Gabriel Looser ein Freund von mir ist; die Idee zum vorliegenden Beitrag stammt jedoch von mir.

Interessanterweise halten wir modernen westlichen Menschen uns in unserer Denkweise meistens für «aufgeklärt», offen und fortschrittlich, obschon wir - kollektiv gesehen geistig eher «verdunkelt» und oft ziemlich eingeengt sind. Besonders deutlich zeigt sich dieser Sachverhalt in unserem kollektiven Denken über den Tod und in unserem konkreten Umgang mit demselben. Als medizinisch Tätige bewegen wir uns in unserer Arbeit oft im Grenzgebiet zwischen Leben und Tod. Um diesen menschlich hochanspruchsvollen Aufgaben mit einer klareren inneren Haltung zu begegnen, brauchen wir aber vorerst für uns selber geeignete, alltagspraxis-taugliche Konzepte zu diesen Themen.
Ein solches Konzept beschreibt Gabriel Looser in seinem Buch. Er betont darin die grosse Bedeutung von Toleranz und nicht urteilendem Respekt anderen Meinungen oder Konzepten gegenüber. Diese Haltung setzt eine eigene Position zwar voraus, wertet diese aber nie im Vergleich mit anderen Positionen als höherstehend ein. Im ganzen Buch wird diese persönliche Haltung spürbar, auch zwischen den Zeilen.

Der Autor lässt uns als Leser anhand eindrucksvoller Beispiele konkret Einblick nehmen in seine Erfahrungswelt als Begleiter vieler Sterbender und deren Angehörigen. Er beschreibt mit behutsamen, aber klaren Worten die Notwendigkeit eines grundsätzlich anderen Bewusstseins und damit einer gelasseneren Haltung gegenüber den Themen Sterben und Tod: «Der Tod ist die logische Folge unserer Geburt und damit keineswegs die grosse Katastrophe, wie unsere Kultur meint und behauptet.» Dabei sind Inhalt und Form der Beschreibungen zwar immer persönlich, bleiben aber sachlich, ohne abzuheben in religiöse Dogmatik und/ oder esoterisch verbrämte Schwärmereien.

Ein ausführliches Kapitel über Organtransplantationen enthält viele nachdenklich stimmende Informationen und beeindruckende persönliche Erfahrungsberichte von direkt Betroffenen und deren Angehörigen. Der Autor schreibt durchaus kritisch: «Der heutige Transplantationseifer ist eine direkte Folge der gigantischen Todesverdrängung unserer gegenwärtigen Kultur.» Weitere Kapitel beschäftigen sich differenziert und sensibel mit den Themen Abtreibung und Sterbehilfe. Selbstverständlich sind auch wir medizinisch Tätigen frei, den Tod - von uns selbst und/oder anderen - zu verdrängen, denn «Sterben ist und bleibt für uns ein Geheimnis - bis wir einst uns selber anschicken, diesen Schritt zu vollziehen». Unsere Rationalität ist damit schlicht überfordert. Dennoch gibt es Möglichkeiten, die uns dabei helfen, den Prozess des Sterbens (und des Lebens!) etwas besser und tiefer zu verstehen. Die Bücher von Gabriel Looser sind nicht nur reiche Informationsquellen dazu, sondern auch Mutmacher, um im oft heiklen Grenzgebiet unserer Arbeit innerlich sicherer und gelassener zu werden.

Weitere Informationen unter: www.institutlooser.ch

Dr. med. Kurt Bettler, Herzogenbuchsee
Notfallmedizin

Felix Schürch

Notfälle in der Hausarztpraxis.

Von Allergie bis Zeckenbiss.

Bern: Hans Huber; 2010.

63 Seiten. Fr. 29.90

ISBN 978-3-456-84778-8

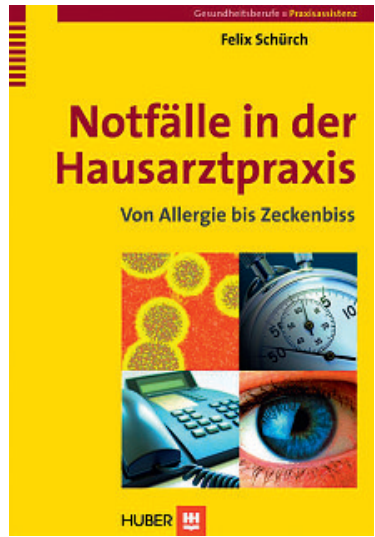

Mit «Notfälle in der Hausarztpraxis» liegt uns ein sehr praktisches Hilfsmittel für Lehrpersonen und Lernende der Medizinischen ArztPraxisassistenz vor. Aus der Fülle des Möglichen muss sich ein Autor immer auf einige bestimmte Themen beschränken, und dies erscheint hier im Hinblick auf die Zielgruppe sehr gut gelungen. Die Kapitel sind übersichtlich, und die konsequente Gliederung erleichtert es, sich innerhalb der verschiedenen Themen rasch zurechtzufinden. Die erläuternden Texte sind verständlich und veranschaulichen die jeweils beschriebene Problematik hervorragend. Gleichzeitig sind sie manchenorts so unterhaltsam geschrieben, dass man immer wieder zum Schmunzeln animiert wird.

Die rasante Entwicklung der Kenntnisse in der Medizin ist eine grosse Herausforderung nicht zuletzt für die Verfasser solcher Lehrmittel und verpflichtet sie zu kontinuierlicher Überarbeitung ihrer Publikationen. Gleichzeitig erinnert dieses Werk die Notfall-Akteure jeder Stufe, ihr Wissen à jour zu halten und zusammen im Team regelmässig Notfallabläufe - zum Beispiel anhand dieses Leitfadens - in der eigenen Praxis zu üben und einzuspielen. So werden schliesslich die nötigen Handgriffe «blind sitzen», alle in der Arztpraxis werden wissen, wo die Notfallmedikamente und -instrumente $\mathrm{zu}$ finden sind, und wie man einander in einer konkreten Situation unterstützen kann. Müsste man allerdings einmal etwas dringend in dem Büchlein nachschlagen, so vermisste man darin einen eigentlichen alphabetischen Index. Dennoch ist es im praktischen Alltag eine gute Hilfe und findet hoffentlich weite Verbreitung.

Dr. med. Frank Wyler-Brem, Projektleiter www.notfallwalkin.ch 